\title{
Neosensitization to Multiple Drugs Following Valproate-Induced Drug Reaction with Eosinophilia and Systemic Symptoms Syndrome
}

\author{
Jae Min Song1, Young Eun Jung², Joon Hyuk Park², Moon Doo Kim², Min Seok Cheon ${ }^{3}$, and Chang In Lee ${ }^{2 凶}$ \\ ${ }^{1}$ Department of Psychiatry, Jeju National University Hospital, Jeju, Republic of Korea \\ ${ }^{2}$ Department of Psychiatry, School of Medicine, Jeju National University, Jeju, Republic of Korea \\ ${ }^{3}$ Department of Dermatology, School of Medicine, Jeju National University, Jeju, Republic of Korea
}

\begin{abstract}
Drug Reaction with Eosinophilia and Systemic Symptoms (DRESS) syndrome is associated with severe skin eruptions, fever, hematological abnormalities, and multi-organ involvement. Although aromatic anticonvulsant drugs have been frequently associated with the manifestation of DRESS syndrome, its induction following treatment with nonaromatic anticonvulsants, such as valproate, has rarely been reported. Moreover, there are limited data regarding the development of neosensitization related to chemically unrelated drugs following an episode of DRESS syndrome. Here, a case of neosensitization to multiple drugs is described. The present case report describes a female patient who experienced neosensitization to amoxicillin, olanzapine, and quetiapine following the manifestation of DRESS syndrome induced by valproate.

Psychiatry Investig 2017;14(4):518-520
\end{abstract}

Key Words DRESS, Neosensitization, Valproate, Amoxicillin, Olanzapine, Quetiapine

\section{INTRODUCTION}

Drug Reaction with Eosinophilia and Systemic Symptoms (DRESS) syndrome is a rare and potentially life-threatening adverse drug-induced reaction. ${ }^{1}$ This syndrome is associated with severe skin eruptions, fever, hematological abnormalities such as eosinophilia or atypical lymphocytes, and multiorgan involvement, especially hepatitis, pneumonitis, nephritis, and lymph node enlargement. ${ }^{1-3}$ DRESS syndrome is also known as Anticonvulsant Hypersensitivity Syndrome (AHS) because treatment with aromatic anticonvulsants such as phenytoin, phenobarbital, and carbamazepine frequently result in the manifestation of these symptoms. ${ }^{4}$ However, DRESS syndrome due to treatment with nonaromatic anticonvulsants, such as valproate, has rarely been reported. ${ }^{5,6}$ The precise physiopathology associated with DRESS syndrome remains uncertain, but a proposed multifactorial model has suggested that the potential causal factors include specific drugs (aromatic

Received: June 23, 2016 Revised: August 26, 2016

Accepted: September 8, 2016 Available online: March 14, 2017

$\triangle$ Correspondence: Chang In Lee, MD, PhD

Department of Psychiatry, School of Medicine, Jeju National University, Aran 13-gil, Jeju 63241, Republic of Korea

Tel: +82-64-717-1530, Fax: +82-64-717-1849, E-mail: handoll@jejunu.ac.kr

(c) This is an Open Access article distributed under the terms of the Creative Commons Attribution Non-Commercial License (http://creativecommons.org/licenses/bync/4.0) which permits unrestricted non-commercial use, distribution, and reproduction in any medium, provided the original work is properly cited. anticonvulsants), reactive drug metabolites, oxidizing stressors, viruses, radiotherapy, sunburn, antioxidant defense system, immunological factors, and concurrent drugs. ${ }^{7}$

The cross-reactivity between anticonvulsant drugs may be explained by chemical or antigenic similarities and has been well described. ${ }^{8}$ However, few studies have reported the recurrence of drug hypersensitivity to chemically unrelated drugs in patients with a history of DRESS syndrome. ${ }^{910}$ The present case report describes a patient who developed secondary neosensitization to amoxicillin, olanzapine, and quetiapine after experiencing DRESS syndrome due to valproate treatment. Neosensitization to multiple drugs is rare, particularly after valproate-induced DRESS syndrome, and no such cases have previously been reported in Korea.

\section{CASE}

A 50-year-old woman with a 15-year history of schizophrenia was being treated with lithium $(1200 \mathrm{mg})$ and quetiapine $(600 \mathrm{mg})$ about 1 month, but due to high lithium serum concentrations, the lithium was changed to valproate $(600 \mathrm{mg})$. Seven days later, the patient developed a whole-body skin rash, facial edema, and hyperthermia. Laboratory tests revealed an abnormal white cell count $\left(25.2 \times 10^{3} / \mu \mathrm{L}\right.$ with $6 \%$ eosinophils) and aspartate transaminase (AST) and alanine transaminase (ALT) concentrations of $2729 \mathrm{IU} / \mathrm{L}$ and 2749 
IU/L, respectively. At that time, the patient had no any other medical history including drug allergy.

A diagnosis of DRESS syndrome due to valproate treatment was established by a consulting dermatologist. And The patient met the original criteria ${ }^{11}$ to established the diagnosis of DRESS syndrome. As a result, all medicines were discontinued because of severe hepatitis, and intravenous methylprednisolone (60 mg per day) was administered for 1 week. The skin rash, fever, and liver dysfunction progressively disappeared. After discharge, the patient was treated with quetiapine $(200 \mathrm{mg})$. However, she became lost to follow up after 6 months.

Approximately 3 years later, the patient was admitted to a local hospital for psychotic symptoms aggravation because she was not taken antipsychotics for 3 years. Therefore, she treated with lithium $(900 \mathrm{mg})$, sulpiride $(600 \mathrm{mg})$, risperidone (2 mg), and quetiapine (100 mg) for 2 weeks. Additionally, the patient initiated treatment with amoxicillin for acute tonsillitis. On the first day of amoxicillin intake, she developed fever, diffuse erythematous macules on her trunk, and facial edema, and she was transferred to a general hospital via the emergency department. Laboratory findings revealed leukocytosis $\left(21.6 \times 10^{3} / \mu \mathrm{L}\right)$ and eosinophilia $(7.8 \%)$ with normal hepatic function. After hospitalization, all medicines, including amoxicillin, were discontinued because hypersensitivity symptoms had a potential to be worsen by lithum, risperidone, and quetiapine, and intramuscular dexamethasone (7.5 mg per day) was administered for 1 week, 4 days later, the patient's psychotic symptoms, including auditory hallucinations and persecutory delusions, were aggravated. Olanzapine $(10 \mathrm{mg})$, which the patient had not been previously administered, was given, but her hypersensitivity symptoms, including fever, skin rash, and abnormal hepatic function, worsened 3 days later. Accordingly, the olanzapine was replaced by haloperidol, and the patient's hypersensitivity symptoms subsided 2 weeks later.

Due to the patient's aggravated psychotic symptoms in spite of administration of haloperidol for 1 month, haloperidol was replaced with quetiapine because she had previously taken it without experiencing hypersensitivity 3 years ago. However, she developed progressive fever and skin rash on the first day of quetiapine intake, and her laboratory tests revealed abnormal hepatic function (AST and ALT concentrations of $78 \mathrm{IU} / \mathrm{L}$ and $79 \mathrm{IU} / \mathrm{L}$, respectively). As a result, the quetiapine was immediately discontinued, and dexamethasone and antihistamine were administered, and her hypersensitivity symptoms gradually improved. To control her psychotic symptoms, the patient began amisulpride treatment, which had not been previously prescribed. Within 2 months, the patient's psychotic symptoms had gradually de- creased and ultimately remitted.

\section{DISCUSSION}

The present case report describes a female patient who experienced neosensitization to amoxicillin, olanzapine, and quetiapine following the manifestation of DRESS syndrome induced by valproate. The patient met the original criteria proposed by Bocquet et al. ${ }^{11}$ to established the diagnosis of DRESS syndrome, which include the following: 1) drug eruption; 2) hematologic abnormalities (i.e., eosinophilia $>1.5 \times 10^{9}$ / $\mathrm{L}$ and the presence of atypical lymphocytes); and 3) systemic manifestations (i.e., adenopathy with lymph nodes $>2 \mathrm{~cm}$; hepatitis with transaminase levels twice the normal values; interstitial nephritis; pneumonitis, and carditis). The patient developed skin eruption after valproate treatment and laboratory findings showed eosinophilia and abnormal hepatic function. Initially, she was diagnosed with DRESS syndrome.

Three years after her treatment with valproate, the patient exhibited a novel hypersensitive reaction to amoxicillin and then developed hypersensitivity to olanzapine and quetiapine after the discontinuation of amoxicillin. Thus, the patient exhibited neosensitization to different types of drugs. She had never been administered olanzapine and had previously taken quetiapine without hypersensitivity. These findings suggest that it is possible to develop neosensitization to multiple drugs after DRESS syndrome.

There have been several reports of cross-reactivity between anticonvulsant drugs during a DRESS episode, and almost all of these cases (40-80\% of patients) included aromatic anticonvulsants such as phenytoin, phenobarbital, and carbamazepine. ${ }^{12}$ Recent studies have reported cross-reactivity between aromatic and non-aromatic anticonvulsants as well..$^{13}$ Additionally, several clinical reports have described neosensitization to chemically and structurally unrelated drugs that were concurrently administered during a previous DRESS episode that was induced by an anticonvulsant. ${ }^{10}$ Only one case report has described a possible hypersensitivity reaction to amoxicillinallopurinol in which amoxicillin was not administered during the first allopurinol-induced DRESS episode. ${ }^{14}$ In the present case, amoxicillin was not administered during the first valproate-induced DRESS episode, but a new hypersensitivity reaction to amoxicillin occurred 3 years later. This suggests that the initial DRESS episode may have caused a massive nonspecific activation of the immune system that resulted in the enhanced expression of co-stimulatory molecules and pro-inflammatory cytokines. The latter allows for a more efficient presentation of chemical antigens to antigen-presenting cells which, in turn, reduces one's tolerance level to drugs, especially antibiotics such as amoxicillin..$^{10,14}$ 
In the present case, neosensitization to amoxicillin, olanzapine, and quetiapine occurred at the same time during the secondary hypersensitivity reaction to amoxicillin. It has been suggested that a transient state of immunosuppression is induced during the hypersensitivity reaction that can trigger latent virus reactivation and a massive nonspecific immune system response, which may in turn lead to the breakdown of tolerance to other drugs that are administered concurrently. ${ }^{10}$ The neosensitization to the administered antipsychotics in the present case could be explained by the structural and chemical similarities of the drugs, but the hypersensitivity reaction was induced by olanzapine and quetiapine rather than amisulpride. Olanzapine and quetiapine have structural and chemical similarities, but these features differ from those of amisulpride.

To our knowledge, this is the first case report of neosensitization to multiple drugs after valproate-induced DRESS syndrome. A thorough search of Pubmed was performed to identify similar cases, which confirmed that no cases of hypersensitivity to amoxicillin or neosensitization to multiple drugs after a valproate-related DRESS episode have been reported. Furthermore, only two studies have reported possible neosensitization to amoxicillin following DRESS episodes induced by carbamazepine, and only one case reported neosensitization to amoxicillin following a DRESS episode induced by allopurinol. ${ }^{10,14}$

There are a number of limitations to our report. First, intradermal patch test to amoxicillin or lymphocyte transformation tests were not performed in this case. Intradermal patch test may be helpful to confirm the culprit drug and identify whether the patient could or could not tolerate drugs. Second, serum immunoglobulin levels was not tested to determine whether immunosuppressive condition. A decrease in immunoglobulin levels might be a predictor of the development of DRESS syndrome.

The present findings suggest that clinicians should be aware of a patient's drug history and exercise caution when prescribing amoxicillin or novel drugs to a patient with a previous DRESS episode.

\section{REFERENCES}

1. Cacoub P, Musette P, Descamps V, Meyer O, Speirs C, Finzi L, et al. The DRESS syndrome: a literature review. Am J Med 2011;124:588-597.

2. Husain Z, Reddy BY, Schwartz RA. DRESS syndrome: Part I. Clinical perspectives. J Am Acad Dermatol 2013;68:693. e691-693.

3. Nanau RM, Neuman MG. Adverse drug reactions induced by valproic acid. Clin Biochem 2013;46:1323-1338.

4. Shear NH, Spielberg SP. Anticonvulsant hypersensitivity syndrome. In vitro assessment of risk. J Clin Invest 1988;82:1826-1832.

5. Huang YL, Hong HS, Wang ZW, Kuo TT. Fatal sodium valproate-induced hypersensitivity syndrome with lichenoid dermatitis and fulminant hepatitis. J Am Acad Dermatol 2003;49:316-319.

6. Roepke S, Treudler R, Anghelescu I, Orfanos CE, Tebbe B. Valproic acid and hypersensitivity syndrome. Am J Psychiatry 2004;161:579.

7. Sullivan JR, Shear NH. The drug hypersensitivity syndrome: what is the pathogenesis? Arch Dermatol 2001;137:357-364.

8. Aouam K, Fredj Nadia B, Amel C, Naceur B. Amoxicillin-induced hypersensitivity after DRESS to carbamazepine. World Allergy Organ J 2010;3:220-222.

9. Aihara Y, Ito S, Aihara M, Kobayashi Y, Yokota S. Different patterns of cytokines, ECP and immunoglobulin profiles at two adverse drug reactions in a patient. Pediatr Int 2005;47:616-621.

10. Gaig P, Garcia-Ortega P, Baltasar M, Bartra J. Drug neosensitization during anticonvulsant hypersensitivity syndrome. J Investig Allergol Clin Immunol 2006;16:321-326.

11. Callot V, Roujeau JC, Bagot M, Wechsler J, Chosidow O, Souteyrand P, et al. Drug-induced pseudolymphoma and hypersensitivity syndrome: Two different clinical entities. Arch Dermatol 1996;132:1315-1321.

12. Hirsch LJ, Arif H, Nahm EA, Buchsbaum R, Resor S Jr, Bazil CW. Cross-sensitivity of skin rashes with antiepileptic drug use. Neurology 2008;71:1527-1534.

13. Aouam K, Ben Romdhane F, Loussaief C, Salem R, Toumi A, Belhadjali H, et al. Hypersensitivity Syndrome Induced by Anticonvulsants: Possible Cross-Reactivity Between Carbamazepine and Lamotrigine. J Clin Pharmacol 2009;49:1488-1491.

14. Ben Fredj N, Aouam K, Chaabane A, Toumi A, Ben Rhomdhane F, Boughattas N, et al. Hypersensitivity to amoxicillin after drug rash with eosinophilia and systemic symptoms (DRESS) to carbamazepine and allopurinol: a possible co-sensitization. Br J Clin Pharmacol 2010;70: 273-276. 